tralian make in particular was almost completely insensitive to cross-nap, while another make, an English one, had a tendency to reverse the napeffect, taking more bias against the nap and less with it. Measurements on these and other bowls with a dial micrometer showed that the less nap-sensitive was the bowl, the more closely it approximated to a spherical form along its actual running-contact tread.

Conversely, the bowls which were most affected, sometimes fantastically, were found to have a radius of curvature across the tread which was much greater than the radius along the tread; for example, a 5 in. bowl, $2 \frac{1}{2}$ in. along the tread, might be $2 \frac{5}{8}$ in. across the tread. The running tread is only about an inch wide, so that such a bowl could be made spherical-treaded by removing only about 0.005 in. at the most. Steps were taken to correct the treads on a set of bowls which were badly affected, but since the re-shaping depended on cutting to limits well inside 0.001 in., it looked like a tool-maker's job to centre each bowl correctly in a lathe. This was practically impossible, when some fifty sets, and more, of club bowls were concerned. It was found, however, that a simple cradle arrangement over a mandrel, with a ball-bearing back-stop and lateral shims between bowl and cradle, gave sufficient drive to allow the use of a scraper. Several sets of bowls were thus converted, taking about half an hour per bowl; they were picked out of the cradle at intervals and tested across the track to a gauge of the same curvature as that which would fit along the track. The lathe was replaced by a sewing-machine stand, and the demand for re-shaping in the Gezira Sporting Club exceeds the supply of willing skilled operatives.

The cause of the reaction of the bowl to the nap is at present uncertain, just as is the causation of the nap itself. But observations show that bowls running across the nap show a marked tilt of their axis of rotation, which may be deflected from the horizontal by as much as $20^{\circ}$. The axis is tilted up in the direction of the nap, just as if the pile of the grass were acting as a banking, and the bowl were running on the banking. We suspect that the effect on the bias is due to this tilting of the axis of rotation (see accompanying diagram).
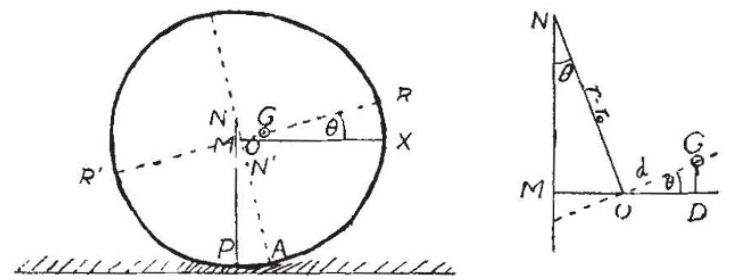

SECTION ACROSS TRACK, BIAS BEING SET WITH THE NAP.

In the above figure, $P$ is the centre of pressure on the grass, but owing to nap the point of maximum grip on the grass is at $A$, hence the tilt of the axis of rotation.

$O$, centre of bowl ; $G$, centre of gravity ; $N$, centre of curvature of section at $P-A ; P N$, perpendicular to the ground at $P ; O M$, perpendicular from $O$ on $P N ; \theta$, angle of slope $=\angle M N O=\angle R O X ; R O R^{\prime}$, axis of rotation; $O A=r_{0} ; N P=r=N A ; \therefore$ $O N=r-r_{0} ; O G=d$.

$\therefore M O D=M O+O D=\left(r-r_{0}\right) \sin \theta+d \cos \theta$.

$\therefore$ increase of bias distance $=M D-O G$, which is equal to $\left(r-r_{0}\right) \sin \theta-d(1-\cos \theta)$.

For $\theta=20^{\circ}, \sin \theta=0.342$, and $1-\cos \theta=0.060$.
Since $d$ is of the order $0 \cdot 1$ in., then $d(1-\cos \theta)$ is of the order $0.006 \mathrm{in.}$, and so may be neglected in comparison with $\left(r-r_{0}\right) \sin \theta$, unless $r=r_{0}$.

The inclination of the spherical bowl is such as to set its median plane at right angles to the slope of the grass blades, in both directions and on both 'hands'. We omit any consideration of gyroscopic effects, which must exist at the beginning of the run of a bowl. Apart from this, the causes of bias are three-fold: first, the gravitational cause due to lateral asymmetry of the bowl; secondly, the shape cause, as exaggerated in the case of a conical 'bowl' ; thirdly, the asymmetry of the lawn structure, as described above. English bowling-greens seem to be free from the latter complication; casual examination seems to indicate that similar one-sided growth happens in our English grasses and climate, but that it is random and localized to an inch or so, the end result being practical symmetry of grass-surface.

We hope that this communication may direct the attention of plant physiologists to an interesting problem, both in the comparison of Cynodon with other grasses, and in the comparison of varied ecological conditions on various grasses. We also hope that it may save some 'greens stewards' being blamed for levelling errors which have no existence.

\section{ACCELERATION OF CHARGED PARTICLES}

1 'HE two most successful methods for accelerating charged particles to very high energies are those employed in the cyclotron and betatron. In the eyclotron, if a very large number of individual accelerations is required, there may be difficulty in keeping the particles in step with the applied oscillating electric field, especially when relativistic mass change causes an appreciable variation in the angular velocity of the particles.

E. M. McMillan (Phys. Rev., 68, 143; 1945) has proposed a device, which because of the similarity in its behaviour to that of a synchronous motor, he has called a 'synchroton'. It is shown that a particle, the energy of which (called its 'equilibrium energy') is such that its angular velocity matches the frequency of the electric field, moves in a stable stationary orbit, and any displaced orbit, due to displacement in phase or energy of the particle, will tend to correct itself. In order to accelerate the particles, the value of the equilibrium energy must be changed by varying either the magnetic field or the frequency. The practical application of the method depends on the type of particles to be accelerated. In the case of electrons, the equilibrium value of the energy varies during the acceleration by a large factor and it is more practical to vary the magnetic field; for heavy-particle acceleration, the frequency may be varied. A possible design for such a $300-\mathrm{MeV}$. accelerator is outlined, and the construction at the Radiation Laboratory of the University of California at Berkeley is stated to have been planned.

D. W. Kerst (Phys. Rev., 68, 233 ; 1945) has also outlined a method for increasing the energies of particles obtained by the betatron. The betatrons used to date have all followed the design of the original 1941 Kerst model (see Nature, January 26, p. 90), in which the flux density at the orbit of the electron is limited to a value less than half the flux density which can be used at the centre of the orbit 
(that is, about 4,000 gauss at the orbit and about 11,000 gauss within the orbit). In the conventional design the flux linking the orbit and the field at the orbit were zero together. This need not be so; the flux linking the orbit can be biased reversely, so that the flux through the centre has a large negative value initially and changes to a large positive value during the acceleration process. Thus the flux density can be made to change by twice the conventional amount, with corresponding increase in the final energy of the electrons. A method of achieving this bias is described, and ways of overcoming difficulties in varia. tion of the radius of the orbit during acceleration as a result of non-linearity of the magnetic characteristics of the iron are discussed.

Modifications in the design along these lines for a betatron of $250 \mathrm{MeV}$. were considered early in 1942 and the plans for construction at the University of Illinois were drawn up during the summer of 1945 . Information received from Germany indicates that a similar plan for a $200 \mathrm{MeV}$. betatron was being drawn up there some time before the capitulation. It has been reported elsewhere that the $250 \mathrm{MeV}$. betatron will be used to investigate high-energy phenomena within the cosmic ray range, and that the cost of the machine and research laboratory will amount to approximately one and a half million dollars.

\section{NATIONAL FRUIT TRIALS}

$I_{\mathrm{s}}^{\mathrm{T}}$ seems appropriate that the Royal Horticultural Society's long-standing assessment of the relative merits of fruit varieties* should be published at a time when a vitamin-conscious but fruit-starved public might be interested in the expansion of Britain's orchards. The maturation of a long-term investigation, planned on sound lines, cannot fail to be an inspiration, and growers should find a considerable number of new varieties which would add more precision to the rather irregular behaviour of many present-day commercial fruit plantations.

The outstanding second-early dessert apple among the new kinds under test is Laxton's Fortune. It is a free and consistent cropper, with good commercial features. Lord Lambourne provides a new midseason apple, but trees are often affected with 'chatfruit' and 'rubbery wood', which reduce its commercial value. For apples stored until April, Winston seems the best of recent introductions, while Laxton's Superb and Sunset might be used instead of Cox's Orange for winter supplies of fruit. Culinary apples do not appear to provide anything now which is superior to Emneth Early, Grenadier or Bramley's Seedling. The advent of Marjorie's Seedling is perhaps outstanding among plums; it provides much better all-round qualities than Monarch, the standard variety for late cropping. It is, moreover, highly resistant to bacterial canker-a strong recommendation in view of the widespread nature of this disease.

The report continues with estimations of varieties of cherries, black currants, gooseberries, red currants, raspberries and strawberries. The two crops last mentioned are scarcely amenable to trial by neighbourly comparison, owing to their different reactions to virus diseases. Juxtaposition of tolerant and susceptible kinds introduces too many variables for experimental proof of varietal worth. Mr. Potter is

* "National Fruit Trials, 1921-1944", by J. M. S. Potter. London : lioyal Horticultural Society, Vincent 'Square, S.W.1. Pp. 56. 5s.,
post paid. alive to these difficulties and to many others. A series of appendixes will be valuable to many workers. Flowering periods of the best varieties of apples, pears and plums are tabulated, and the results of an extensive series of cross-pollinations of the new kinds are given. Sulphur-shy apples and gooseberries are listed. Several apparent synonyms have been found among recent introductions, and these are discussed. Varietal reaction to frost damage has been considered, and it appears that dessert varieties are on the whole less susceptible to this kind of damage than are culinary kinds. Trees grafted upon Malling stocks I and II were more resistant to frost than those workec on Malling type XII. Young trees appeared to be more susceptible than fully grown trees.

Many fruit varieties have been sent to sub-stations in various parts of England, and the behaviour of trees at various places provides the strongest argument for the extension of fruit trials, based upon the sound details of the Wisley scheme. Scotland, in particular, gives a climate altogether different from that at Wisley, and requires its own estimate of varietal worth. The National Fruit Trials have shown what can be achieved; their greatest future lies in geographical extension.

\section{LIVERPOOL OBSERVATORY AND TIDAL INSTITUTE}

$\mathrm{D}$ URING the War, the publication of the annual reports of the Liverpool Observatory and Tidal Institute was discontinued, the last report issued for general circulation being that for 1939. Publication is now resumed with a combined report for the years 1940-45; this also embodies a centenary report on the Liverpool Observatory, which was opened in 1845. The Liverpool Dock Act of 1841 gave powers to the town council to build and maintain a marine observatory out of the dock dues; the early work consisted of time determination (the results being notified to ships in port by a time-ball), meteorological observations and forecasts, and the rating of chronometers. The first director, Hartnup, was a pioneer in showing the principal source of error in chronometers to be variable temperature, and he instituted the now standard tests in chambers at controlled temperatures.

In 1864 the Observatory, by then under the control of the Mersey Docks and Harbour Board, was transferred to fine new buildings at Bidston Hill.

In the early years of the present century, the astronomical work gradually fell into the background, and later wireless telegraphy made the time determinations unnecessary. More recently the meteor. ological work has declined, as the State organization for this purpose has developed. Nevertheless, the present report includes the usual meteorological summaries for each of the years 1940-44. Seismological work, which was started in 1897, has continued, and has a marine interest in connexion with the tilting of the land under the varying load of tidal water.

In 1929 the Observatory and the Tidal Institute, founded in 1919 as a joint enterprise of the University of Liverpool and the Mersey Docks and Harbour Board, were combined, and the flourishing young Institute became housed in the Observatory.

The main part of the report deals with the Institute's many activities during the War, especially 